\title{
One-shot Evaluation of the Control Interface of a Robotic Arm by Non-Experts
}

\author{
Sebastian Marichal ${ }^{1,2 *}$, Adrien Malaisé ${ }^{1 *}$, Valerio Modugno ${ }^{1,3}$, Oriane Dermy ${ }^{1}$, \\ François Charpillet ${ }^{1}$ and Serena Ivaldi ${ }^{1}$ \\ 1 Inria, Villers-lès-Nancy, F-54600, France; Loria, UMR n.7503, CNRS \& Univ. \\ Lorraine, Loria, Vandoeuvre-lès-Nancy, F-54500, France. name.surname@inria.fr \\ 2 Universitat Pompeu Fabra, Barcelona, Spain. sebastian.marichal@upf .edu \\ 3 DIAG, Sapienza University, 00185 Roma, Italy. \\ * equal contribution
}

\begin{abstract}
In this paper we study the relation between the performance of use and user preferences for a robotic arm control interface. We are interested in the user preference of non-experts after a one-shot evaluation of the interfaces on a test task. We also probe into the possible relation between user performance and individual factors. After a focus group study, we choose to compare the robotic arm joystick and a graphical user interface. Then, we studied the user performance and subjective evaluation of the interfaces during an experiment with the robot arm Jaco and $\mathrm{N}=23$ healthy adults. Our preliminary results show that the user preference for a particular interface does not seem to depend on their performance in using it: for example, many users expressed their preference for the joystick while they were better performing with the graphical interface. Contrary to our expectations, this result does not seem to relate to the user's individual factors that we evaluated, namely desire for control and negative attitude towards robots.
\end{abstract}

Keywords: Human-robot interfaces, user evaluation, individual factors, nonexperts

\section{INTRODUCTION}

In this paper, we address the question of the preference for a robotic interface by non-experts (or naive users without training in robotics), after one single evaluation of such an interface on a simple task. This refers to situations when non-experts face the decision of adopting a robot for episodic use (i.e., not a regular continuous use as workers in factories): the ease of use of an interface is crucial for the robot acceptance. We do not target users that could have or will have the time to receive a proper training on how to use a robot. While in manufacturing, robots are used by skilled workers that receive a proper training for operating the robots, this training is not likely to happen for many assistance and service scenarios: for example, inside an healthcare facility it is likely that the nurses or the patients will never receive a proper training for operating and interacting with the robots. The question arises on how to make the robot easily 
controllable by such users and facilitate their interaction with the robot. As the interface for controlling the robot is an essential part of the robotics system, this question impacts not only the interaction performance, but also the user acceptance and final adoption of the technology.

In this study, we focus on the Kinova Jaco (see Fig. 1), a lightweight robotic arm which can be controlled with a built-in joystick. It was designed for a daily and regular use for ordinary people after some training: the joystick is easy to manipulate but it has several buttons and control modes that require practice to achieve a fluent interaction. Here, we target a different use and a one-shot evaluation: if the control interface is an obstacle to the use of the robot, the users will not likely adopt the robot even for sporadic use. Several interfaces for robot control have been investigated in HRI. For example [17] investigated touch, speech and gestures for teaching a robot a nursery rhyme, finding that users do not prefer a particular modality but enjoy less touching the robot. In [16] the authors compared haptic interfaces with buttons, finding that users preferred buttons for simple tasks and physical command for complex tasks requiring high precision. Here, We compare the joystick with a ad-hoc graphical user interface (GUI) with buttons.

We are here interested in (i) probing the

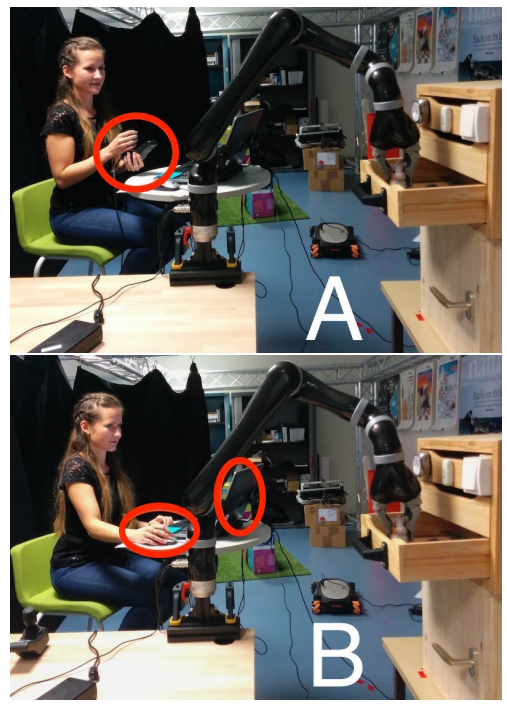

Fig. 1. The experimental setup with the Kinova Jaco arm. The participant moves the arm using (A) the joystick and (B) the graphical interface on the laptop. relation between individual factors and user performances for robot interfaces, and (ii) studying the relation between the performances that the user achieve with such interfaces and their preference.

Our main hypothesis is that the preference of an interface is related to the performance of using it. This premise is evident from other studies focused on interfaces evaluation. Guo \& Sharlin noted that preferences for a tangible interface was related to a stronger performance in using it [15]. Many studies on control interfaces for robots focused on graphical user interfaces for their better acceptance by non-experts, for example [6] for teaching objects to a robot, [4] for applications in rehabilitation and medicine. In [5] the authors proposed an Android interface for moving the Jaco arm, but unfortunately it was not thoroughly evaluated by final users.

Our second hypothesis is that individual factors, such as traits and attitudes, may influence the user performances with the robot interfaces. There is indeed prior evidence that some personality traits have significant effects on the perceived ease of use of new technologies, such as smartphones [7]. There is also 
evidence that personality traits and attitudes have some influence in HRI in the context of social robotics [1]. It seems therefore rational to explore the relation between individual factors and the user perception and performance in controlling a robot. Two attitudes seems particularly relevant for our study: the Negative Attitude towards robots (NARS) [3], which captures the anxiety of an imagined interaction with a robot, and the Desire For Control (DFC) [8], which captures the attitude to be in control or control situations. The first could influence for example the time spent on using the robot, while the second could influence the preference for an interface that provides a stronger sensation of controlling the robot.

Our study was split in two phases. In the first, we carried out a focus group study to identify the main concerns of people interacting with a robotic arm, the key elements underlying their imagined interaction and the imagined interfaces to control the robot movement. This set enabled us to formulate the first hypothesis and choose a graphical user interface (GUI) as an intuitive interface alternative to the Kinova joystick. The second phase concerned the experiments with the Jaco robot and the two interfaces. We first performed a pilot study with University students to test the experimental setup and gain preliminary insights for the later final experiments with ordinary adults. The analysis of the pilot study and the outcome of the focus group enabled us to refine the evaluation questionnaires to be used for the final experiments and formulate new hypothesis.

We studied the user performance and subjective evaluation of the interfaces during an experiment with the robot arm Jaco and $\mathrm{N}=23$ healthy adults. We provide quantitative evidence of the different performances obtained by nonexperts, using both interfaces for the first time to realize some tasks. We also report on the user feedback in using the two interfaces, which provides us useful information to inform future interface designers.

Our preliminary results show that the user preference for a particular interface does not seem to depend on their performance in using it: for example, many users expressed their preference for the joystick whereas they were better performing with the graphical interface. Also, contrary to our expectations, this result does not seem to relate to the user's aforementioned individual factors.

Research Hypothesis - Given the previous results in the literature, we expect that the GUI will be easier to use than the joystick, for non-trained users. The GUI has the advantage to not require too much training, and it provides some graphical shortcuts to the main robot configurations. To provide a quantitative measure of the ease of use, we use the duration of execution of tasks performed with an interface, and the number of errors done while using it. We formulate the hypothesis as:

H1) The time necessary to complete the tasks with the GUI is shorter than with the joystick.

H2) The number of precision errors with the GUI is lower than with the joystick. H3) The number of mapping errors with the GUI is lower than with the joystick. 

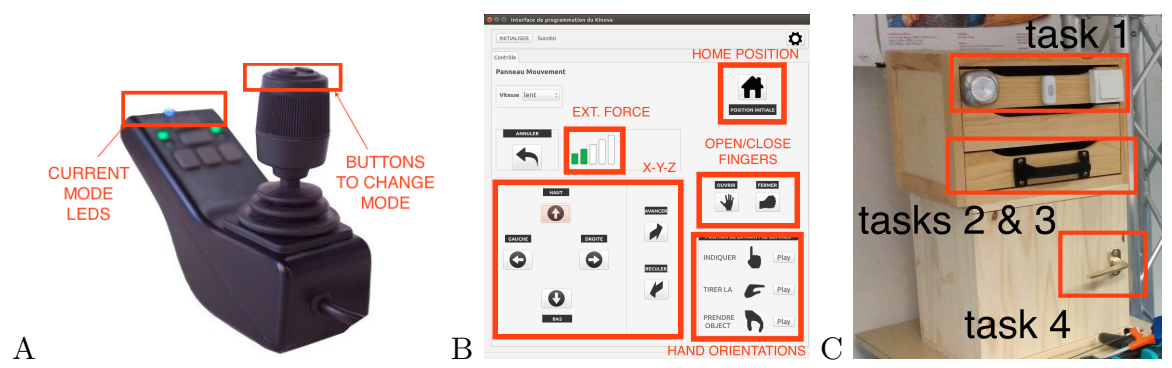

Fig. 2. The two interfaces used for the evaluation: (A) the Kinova joystick and (B) our ad-hoc graphical interface on the laptop. (C) The Activities of Daily Living setup: click the three buttons (task 1), open a drawer (task 2), take an object inside the drawer (task 3), open the door (task 4).

We also hypothesize that the user personality, attitudes and their prior experience with related technologies may influence the user acceptance of the proposed technologies and the performance in using it. The desire for control could play a crucial role in the preference for the joystick to the GUI, as the users could have the impression to be more in control of the robot while moving it. The negative attitude towards robots could influence the user perception of the interaction and the perceived ease of use. We formulate therefore the following hypothesis:

H4) Participants with high score of DFC will prefer the joystick to the GUI.

H5) Participants with a high negative attitude towards robots score will make more errors and have a lower perceived ease of use and user satisfaction.

\section{METHODS}

\section{Participants}

The participants were all French, healthy adults that volunteered to take part in the study. The focus group study was carried out with 6 adults (age: $39.16 \pm$ 15.71, 3 males, 3 females) without or with little robotics experience (1 participant). The pilot study was carried out with 7 University students in cognitive sciences (age: $23.14 \pm 1.46,2$ males, 5 females). The final experiments with the robot were carried out with 23 adults (age: $35.13 \pm 11.98,12$ males, 11 females) without robotics experience.

\section{Experimental setup}

The experiments were carried out at the LARSEN laboratory of INRIA (Nancy, France). The experimental setup was organized as shown in Fig. 1. A desk with a laptop was placed in front of the Kinova Jaco arm, fixed on a table. The arm was positioned in such a way to be able to perform some manipulations on the ADL setup (Fig. 2C), made of two boxes: one with a door handle, one with three buttons and a drawer containing a small object. A video camera, placed 
behind the participants, was used to record the experiments. Two interfaces (see Fig. 2A-B) for controlling the robot were used: the native joystick by Kinova and our own ad-hoc graphical user interface (GUI). The joystick can move the hand in the Cartesian space (position and rotation), open and close the fingers. Two buttons are used to select whether to move the hand position (mode 1), its orientation (mode 2) or the fingers (mode 3). The GUI was developed with Qt and is open-source ${ }^{4}$. Both interfaces use the same Kinova API for robot control and inverse kinematics solving.

\section{Questionnaires}

To probe into the influence of individual factors, we asked the participants to the robot experiment to fill out some questionnaires before the experiments: the Negative Attitude Towards Robots Scale (NARS) [3] and the Desire For Control scale (DFC) [8]. Our French adaptation was used [1]. The participants also filled two post-experimental questionnaires consisting of questions/affirmations adapted from usability and technology acceptance models to a robotic context as it was done in previous works $[12,9]$. The post-block questionnaire, at the end of each experimental condition (block when one interface is used), was based on the USE questionnaire [13] (typical questions were "How good will you rate the movement you achieved in the 'open the drawer' task?"). The post-experimental questionnaire consisted of a set of affirmations to be rated on a 7-points Likert scale, targeting constructs typical of the UTAUT [11] and TAM 3 models [14] (typical questions were "Controlling the robot with the GUI is easy").

\section{Experimental protocol}

The study consist of a focus group and two robot experiments: a pilot study with University students, then experiments with ordinary adults. All the data were recorded in anonymous form through a random numerical id attributed to each participant. All participants were equally informed by the experimenter about the purpose of the study and their rights, according to the ethics guidelines of our institute. An informed consent form was signed by each participant. The protocol received the positive approbation of the local Ethics Committee.

Focus group study - We asked a group of 6 adults without or with little experience in robotics to imagine how they would interact with the robot and control it to do some tasks. The group gathered in a closed room around a table. One moderator led the group, while two recorders took notes and annotated sentences and body language. The session lasted about 2 hours and was recorded for analysis purposes. The experimenter asked to the group six warm-up questions, such as "Tell us about your overall experience with robots", "In which situation(s) do you imagine that a robotic arm such as the Kinova would be useful?". In a work in pairs, participants had to present their ideas about interfaces for controlling a robot arm.

Pilot study with the robot - We carried out a pilot study with the Jaco robot and 7 University students. Each participant had to perform the 4 tasks (see Figure 2C) with the robot, using the joystick and the GUI. The order of the

\footnotetext{
${ }^{4}$ https://github.com/serena-ivaldi/kinova-modules
} 
interfaces was randomized across the participants. After the experiment with the robot, we asked the participant to express their preference for one of the two interfaces and provide their feedback and personal evaluations.

Experimental study with the robot - The experiments with the Jaco robot were carried out with 23 adults without expertise in robotics. Each participant filled in the questionnaires NARS and DFC one week before the experiment. The day of the experiment, the participant was welcomed to the laboratory room by the experimenter and seated on a table with a laptop (see Fig. 1) in front of the robot. There were two blocks corresponding to the two experimental conditions: one with the joystick and one with the GUI. In each block, the participant had to perform the 4 tasks with the robot (see Fig.2C). The order of use of the interface was randomized and balanced across the participants. To ensure that all the participants received an equal set of instructions, we provided them with the same instructions, either in paper format and in video format (tutorial). The participant started by reading some paper instructions explaining the 4 tasks to be performed with the robot. After reading the instructions, they had to rate some statements on a 7-items Likert scale, such as "The required tasks are difficult" and "The instructions were difficult to read". We also added two trick questions to check if they were attentive and had carefully read the instructions. Before each block, the participant watched a $2 / 3$ minutes video tutorial explaining how to use each interface, then he/she could familiarize and try it for about 1 minute. We instructed the participants to follow a think-aloud protocol. When the participant was ready to start, he/she began performing the 4 tasks in sequence. Two experimenters monitored and annotated the experiment. After completion, the participant filled in a questionnaire evaluating the ease of use of the interface. The sequence tutorial-test-tasks-evaluation was repeated for the second interface. After the experiment with the robot, the participant filled in the post-experimental evaluation questionnaire, then answered to some semi-directed questions during an interview with the two experimenters.

\section{Measures and data analysis}

During the focus group, two recorders annotated the discussion. Video recordings were used to complete the annotation offline. In the pilot study, we measured the duration of each task and the user preference for each interface. In the robot experiments, we employed both objective and subjective measures. Two experimenters annotated: the duration of each task; the numbers of precision errors, represented by the number of times the robot hit the ADL board; the number of mapping errors, represented by the number of times the robot was moved in the opposite direction with respect to the desired (we could identify this by the explicit verbalization of the participant, or by two consecutive movements in opposite directions where the first was clearly in the wrong direction with respect to the goal of the movement). The questionnaires' score for NARS and DFC were computed according to the authors' recommendations. The subjective measures retrieved from the post-experimental questionnaires are the perceived ease of use (PEOU, typical question: "Controlling the robot with the GUI is easy")), the user satisfaction (US, "How good will you rate the movement you 
achieved in the 'open the drawer' task?") and the facilitating condition (FC, "The time to test the Joystick before the experiment was enough") related to each interface, computed by the sum of the score of the questionnaire items for each construct. The expertise in using joysticks was a self-reported score on a 10-item scale.

Unless otherwise stated, we computed median and standard deviation of all the measured variables; we used Spearman's correlation and verified the statistical significance of the different conditions with a Wilcoxon signed ranked test with continuity correction in $\mathrm{R}$.

\section{RESULTS}

Focus group - The focus group participants did not have a particular affinity with robotics, and were generally worried about the possibility of robots replacing humans. When asked about the possible use for the Jaco arm, they indicated grabbing objects on very high shelves, assisting people with impairments or arm troubles, doing manual tasks like laundry, ironing and painting walls. Almost all the participants agreed that the robot should not be completely autonomous: they need to be in control of the situation when the robot is acting. They said that they should "teach the robot to do the things the way we want" and "be able to stop the robot anytime". When we asked how to control the robot, the participants mostly indicated panels with buttons (3/6). In particular, one participant explained that there should be a button for each possible robot gesture.

Pilot study - The only significant difference in terms of task duration with the two interfaces is on the second task (opening the drawer, $\mathrm{V}=0 \mathrm{p}=0.0156<0.05$ ). We did not find any significant correlation between the task duration and the participants' self-report expertise with joysticks.

Concerning the joystick, the negative points were: the difficulty in controlling the hand orientation and the way to change the modes with the buttons. Positive points were that it was more intuitive to move in the $x-y-z$ space, especially for the students used to play video-games, and that it felt like an "extension of their arm". Concerning the GUI, the negative point was that it required to switch continuously the attention from the laptop to the robot. The positive points were its clearer design that made the actions explicit and the ease of use when choosing pre-determined orientations of the hand for manipulation.

We asked the 7 participants to choose the interface that was easier to use and more intuitive for them: 2 preferred the joystick and 5 the GUI ("it can be mastered, one makes more errors with the joystick").

Robot experiments - After reading the instructions, the participants evaluated the tasks to be not difficult (on a 7 -item Likert scale, median $=2$, stdev $=1.67$ ) and the instructions easy to read (median $=1$, stdev $=2.03)$. We found a significant difference in the overall duration of the tasks $(\mathrm{V}=25 \mathrm{p}=0.0006<0.001)$ for the two conditions, in particular for Task 2 (opening the drawer, $\mathrm{V}=10 \mathrm{p}=0.0002<0.001$ ) and Task 3 (grabbing the object, $\mathrm{V}=28.5 \mathrm{p}=0.0009<0.001$ ), a fair difference for Task 4 (opening the door, $\mathrm{V}=51.5 \mathrm{p}=0.0089<0.01$ ). We also compared the dura- 
tion of the tasks executed with each interface when the latter is first or second in order of execution: we did not find difference in the execution for the GUI (Mann-Whitney, $\mathrm{W}=82 \mathrm{p}=0.347$ (N.S.)), whereas there is a weak evidence for a difference in the execution time of the joystick if it is used as first or second (Mann-Whitney, $\mathrm{W}=27.5 \mathrm{p}=0.0193<0.05)$. In terms of use of the interface, there is a marginal difference in terms of precision $(\mathrm{V}=53 \mathrm{p}=0.0531$ (N.S.)), while there is a strong difference in terms of mapping errors $(V=0 \quad p=2.85 e-$ $05<0.001)$ - the median number of mapping errors with the joystick is also quite elevated (10). Regarding the subjective measures retrieved by the questions, we found a significant difference in the ratings in terms of ease of use $(\mathrm{V}=251.5$ $\mathrm{p}=5.23 \mathrm{e}-05<0.001)$, satisfaction $(\mathrm{V}=239 \mathrm{p}=0.0022<0.005)$ and facilitating conditions $(\mathrm{V}=159 \mathrm{p}=0.0013<0.005)$ : the GUI has higher ratings than the joystick on all the three items. We did not find a significant correlation between the users' performance and their prior expertise in using joysticks nor between the user performance and their NARS.

Among the 23 participants, 11 expressed preference for the joystick and 12 for the GUI. However, in terms of usability, the joystick was favored by 6 participants, while the GUI by 16 (one participant said they were equal). We tested if the interface preference was related to the DFC score of the participants but we did not find any significant difference (Mann-Whitney, $\mathrm{W}=48, \mathrm{p}=0.279$ (N.S.))

We asked the participants to provide their feedback in the post-experimental interview. Many participants highlighted that the joystick made them feel more "in control" when moving in the main Cartesian directions (x,y,z - the first mode of the joystick) and that they could achieve more precise movements with it. Almost all the participants reported that switching the mode with the joystick was very difficult. However, some thought that they could become good users with a dedicated training. One participant, for example, said "my son is very good with the video-games pad, he will learn in 10 minutes; for me, I will need some hours". Many participants appreciated the GUI because of the intuitive buttons where each command/action was explicit.

\section{DISCUSSION}

In this study we focused on non-expert users controlling a robot for their first time: if the robot-user does not have a proper training, or if he is using the robot only once in a while, which interface could be easier to use and facilitating the robot adoption? From the focus group study, we learned that people imagine to interact with the robot in a structured way (e.g., buttons) that allows them to be in control of the robot decisions (e.g., when to start, when to stop).

To make the robot controllable by non-experts, our conclusion is that we need a very reliable control interface that they can understand and use easily/intuitively, that is robust and that gives them the impression to be in control. From the participants suggestions, a panel with buttons seems appropriate as a control interface: it gives the user the impression that the robot can act upon their orders. For the purpose of this study, we decided that the most appropriate 
control interface to test against the joystick of the Jaco arm was a GUI with buttons.

Is a GUI really better than a joystick? - From the pilot study with students, we could not strongly conclude that the GUI brings notable improvements over the joystick. In the experiments with ordinary adults, the GUI is better than the joystick in terms of objective performance measures and subjective user evaluation. We found significant difference in the duration of tasks and mapping errors, but not in the precision errors: therefore we accept $\mathbf{H 1}$ and $\mathbf{H 3}$ but reject H2. Almost all participants found the GUI easier to use, more understandable and straightforward. Many participants appreciated moving the robot with the joystick as they felt it an "extension of their hand". Interestingly, while most participants appreciated the pre-programmed orientations/configurations of the hand, that were quite difficult to obtain with the joystick, some participants reported them as a constraint that was limiting their freedom to choose different orientations of the hand to realize the tasks. These participants suggested that the two interfaces should be combined to give the user more freedom. It is however important to notice that the GUI performs better than the joystick in our particular experimental conditions, where the participants have a very limited training for using the interfaces (a video tutorial and 1 minute to familiarize with the interface and try it). The results could be very different in a case where the participant uses the robot on a regular basis or receives a proper training. We will address this case in future experiments.

Do individual factors play a role in the user performance with an interface? - Our preliminary results show that the user preference for a particular interface does not seem to relate to their performance in using it: for example, many users expressed their preference for the joystick whereas they were better with the GUI. Contrary to our expectations, this result does not seem to relate to the user's individual factors, as we did not find a strong evidence to support our hypothesis. We did not find significant correlations between the user preferences or performances with both NARS and DFC. We therefore reject $\mathbf{H} 4$ and H5. Nevertheless, in the post-experimental interviews many participants reported to feel more comfortable with the joystick despite being better with the GUI: this may seem counter-intuitive, but in fact suggests that there may be other individual criteria that drive their choice.

\section{CONCLUSIONS}

Two main questions emerge for future work: Which are the key factors that determine user preference for a robot control interface and if the preference and

performance in using an interface would change in a long term scenario (i.e., a scenario where users receive a training for operating the robot with the interface and use such an interface more frequently or on a daily basis). We plan more experiments to investigate more thoroughly all these questions. 


\section{References}

1. Ivaldi, S.; Lefort, S.; Peters, J.; Chetouani, M.; Provasi, J.; Zibetti, E. (2016) Towards engagement models that consider individual factors in HRI: on the relation of extroversion and negative attitude towards robots to gaze and speech during a human-robot assembly task. Int. Journal of Social Robotics.

2. Gaudiello, I.; Zibetti, E.; Lefort, S.; Chetouani, M.; Ivaldi, S. (2016) Trust as indicator of robot functional and social acceptance. An experimental study on user conformation to the iCub's answers. Computers in Human Behavior.

3. Nomura, T., Kanda, T., \& Suzuki, T. (2006). Experimental investigation into influence of negative attitudes toward robots on human-robot interaction. AI \& Society,

4. Chung, C.S.; Wang, H. \& Cooper, R.A. (2013) Functional assessment and performance evaluation for assistive robotic manipulators: Literature review. J Spinal Cord Med.; 36(4): 273-289

5. Chung, Boninger, Wang \& Cooper. (2015) The Jacontrol: development of a smartphone interface for the assistive robotic manipulator. RESNA Annual Conference.

6. Rouanet, Danieau \& Oudeyer (2011) A robotic game to evaluate interfaces used to show and teach visual objects to a robot in real world condition. Int. Conf. on Human-Robot Interaction, pp. 313-320.

7. Ozbek, et al. (2014) The impact of personality on Technology acceptance: a study on smart phone users. Procedia of Social and Behavioral Sciences 150: 541-551.

8. Burger, J. M., \& Cooper, H. M. (1979). The desirability of Control. Motivation and Emotion, 3, (4), 381-393.

9. BenMessaoud, C.; Kharrazi, H. \& F. MacDorman, K.F. (2011) Facilitators and Barriers to Adopting Robotic-Assisted Surgery: Contextualizing the Unified Theory of Acceptance and Use of Technology. PLoS One, 6(1): e16395.

10. Lee, J.Y.; Choi, J.J. \& Kwak, S.S. (2015) The Impact of User Control Design Types on People's Perception of a Robot. Proc. HRI ext. abstr., p. 19-20.

11. Venkatesh, V., Morris, M.G., Davis, F.D., and Davis, G.B. (2003) User Acceptance of Information Technology: Toward a Unified View, MIS Quarterly, 27, 425-478

12. Heerink, M.; Krse, B.; Wielinga, B. \& Evers, V. (2009) Measuring the influence of social abilities on acceptance of an interface robot and a screen agent by elderly users. Proc. 23rd British HCI Group Annual Conf., pp.430-439.

13. Lund, A.M. (2001) Measuring Usability with the USE Questionnaire. STC Usability SIG Newsletter, 8:2.

14. Venkatesh, V., \& Bala, H. (2008) Technology Acceptance Model 3 and a Research Agenda on Interventions, Decision Sciences, 39, 273-315.

15. Guo, C. \& Sharlin, E. (2008) Exploring the Use of Tangible User Interfaces for Human-Robot Interaction: A Comparative Study. Proc. CHI, p. 121-130.

16. Gleeson, B., Currie, K., MacLean, K. \& Croft, E. (2015) Tap and Push: Assessing the Value of Direct Physical Control in Human-Robot Collaborative Tasks. J. of Human-Robot Interaction, 4(1):95-113.

17. Novanda, O., Salem, M., Saunders, J., Walters, M.L. \& Dautenhahn, K. (2016) What Communication Modalities Do Users Prefer in Real Time HRI?, 5th Int. Symposium on New Frontiers in HRI 2016. 